\title{
Note on the robustness of the neutrino mass bounds from cosmology
}

\author{
Robert H. Brandenberger* \\ Department of Physics, Brown University, Providence, Rhode Island 02912, USA \\ and Perimeter Institute for Theoretical Physics, Waterloo, Canada N2J 2 W9 \\ Anupam Mazumdar ${ }^{\dagger}$ \\ Department of Physics, McGill University, Montréal, Québec, Canada H3A $2 T 8$ \\ Masahide Yamaguchi ${ }^{\ddagger}$ \\ Department of Physics, Brown University, Providence, Rhode Island 02912, USA
}

(Received 29 January 2004; published 16 April 2004)

\begin{abstract}
The recent high precision maps of cosmic microwave anisotropies combined with measurements of the galaxy power spectrum from new large-scale redshift surveys have allowed stringent bounds on the sum of the neutrino masses to be placed. The past analyses, however, have implicitly assumed that the spectrum of primordial density fluctuations is adiabatic and coherent, as predicted in the simplest models of inflation. In this paper, we show that the limits hold even if the assumption on the primordial power spectrum is relaxed to allow for a contribution of nonadiabatic, incoherent fluctuations such as would be predicted by topological defects.
\end{abstract}

DOI: 10.1103/PhysRevD.69.081301

PACS number(s): $98.80 . \mathrm{Cq}$

\section{INTRODUCTION}

Recently, there has been a lot of progress in neutrino physics. Measurements of solar and atmospheric neutrino fluxes have yielded tight bounds on the mass differences between the different neutrino mass eigenstates (see e.g. [1] for recent reviews). The bounds on the absolute masses from direct measurements, however, are much weaker. Currently, the bounds on the absolute neutrino masses coming from indirect cosmological considerations are much stronger. In particular, given the recent high precision data on cosmic microwave background (CMB) anisotropies and the increased information about the galaxy power spectrum based on the recently completed large-scale galaxy redshift surveys, it is possible to deduce tight bounds on the sum of the neutrino masses [2]. These analyses, however, make use of certain assumptions about the evolution of the early Universe. Specifically, they make use of theoretical predictions for the power spectra of the galaxy distribution, derived under the assumption that the primordial spectrum of cosmological perturbations was purely adiabatic and coherent, as predicted in simple single field models of inflation. In this paper we show that the bounds derived in [2] are robust against the addition of a contribution to the primordial power spectrum which is nonadiabatic and incoherent, namely a contribution coming from a distribution of topological defects.

The cosmological limits on the sum of the neutrino masses are derived by combining the high precision data of $\mathrm{CMB}$ anisotropies with the measurements of the galaxy power spectrum which have recently improved in accuracy

\footnotetext{
*Email address: rhb@het.brown.edu

†Email address: anupamm@hep.physics.mcgill.ca

ॠEmail address: gucci@het.brown.edu
}

upon completion of the $2 \mathrm{dF}$ (two degree field) large scale structure survey. Given the assumption that the primordial power spectrum is purely adiabatic and coherent (as predicted in simple single scalar field models of inflation), the CMB angular power spectrum determines to good accuracy the slope of the primordial power spectrum and the cosmological parameters (see e.g. [3] for recent reviews). The most sensitive dependence on the neutrino masses comes from the smallest scales, those which are not (yet) probed by CMB anisotropies but rather by the galaxy power spectrum. The reason for this dependence lies in the phenomenon of neutrino free streaming [4]. Because of their small mass, the neutrinos have large velocities at $t_{e q}$, the time of equal matter and radiation. Hence, neutrino density perturbations on comoving scales smaller than the neutrino free streaming length (the distance the neutrinos travel in one Hubble expansion time) at $t_{e q}$ are suppressed (the suppression factor increases exponentially as a function of the wave number). Hence, if the contribution of the neutrinos to $\Omega$ (the energy density in units of the critical density) is too large, there will be insufficient power to explain the observed magnitude of the galaxy power spectrum. A lower bound on the galaxy power spectrum thus translates into an upper bound on the contribution of neutrinos to $\Omega$, and thus to an upper bound on the sum of the neutrino masses. Note that the most stringent constraints come from the smallest length scales for which the galaxy power spectrum can be reliably determined.

However, the assumption that the primordial spectrum of fluctuations is purely adiabatic and coherent is a very restrictive one. Even in the context of scalar field driven inflationary cosmology, as soon as one considers models with more than one scalar field, it is possible to obtain a contribution of entropy fluctuations [5]. Many inflationary models based on grand unified theories (see e.g. [6]) or on the brane inflation scenario (see e.g. [7]) predict a contribution to the power 
spectrum from cosmic strings, yielding a contribution of isocurvature and incoherent fluctuations. It has been known for a long time $[8,9]$ that the transfer function which relates the primordial power spectrum to the present power spectrum changes dramatically on small distance scales if a contribution of seed perturbations such as those created by cosmic strings is added. The basic point is that the cosmic strings constitute density fluctuations seeds which are not erased by neutrino free streaming. Thus, in a seed model, the accretion of neutrinos on small scales is delayed (and thus reduced in amplitude) but not prevented. Hence, it appears at first sight that even a small addition of cosmic string seed fluctuations to the primordial power spectrum might dramatically loosen the cosmological bounds on the sum of the neutrino masses. Here, we shall demonstrate that this is in fact not the case.

The outline of our analysis is as follows. We will first consider the largest contribution of cosmic strings to the angular power spectrum on large angular scales which is consistent with the current data on CMB anisotropies, making use of the recent Wilkinson Microwave Anistropy Probe (WMAP) results [10]. This result will then determine the contribution of the cosmic strings to the mass power spectrum. In this context, it is important to take into account the fact that the perturbations produced by the strings are isocurvature in nature (as emphasized e.g. in [11]). This will effect the ratio of their contributions to the angular $\mathrm{CMB}$ power spectrum and the matter power spectrum. We then use the (approximately) known transfer function for strings and cold dark matter (which is larger on small scales than the standard transfer function for adiabatic fluctuations with cold dark matter) to estimate the total matter power spectrum on small scales, and analyze whether the loss of power on small scales when increasing the neutrino contribution to $\Omega$ can be compensated by the increase in power coming from the presence of cosmic string seed perturbations.

\section{ANALYSIS}

The recent WMAP data on the angular power spectrum of CMB anisotropies has mapped out with high precision the region of the power spectrum corresponding to the first acoustic peak. This peak is narrowly centered at a value $l$ $=220 \pm 1$ [10], in good agreement with a cosmology in which the Universe is spatially flat and the primordial spectrum of fluctuations is coherent and adiabatic. Cosmic strings, on the other hand, give rise to isocurvature fluctuations which are incoherent. As a consequence, there are no marked acoustic oscillations in the angular power spectrum [12-15], but only a fairly broad Doppler peak [16]. Thus, the present data tightly constrain the maximal contribution of strings to the angular power spectrum of $\mathrm{CMB}$ anisotropies on large angular scales.

Since the theoretical predictions for the spectrum of CMB anisotropies resulting from cosmic strings in the acoustic peak region are quite uncertain, the bounds on the contribution of cosmic strings to the $\mathrm{CMB}$ angular power spectrum on large scales are also not certain. Different investigations have yielded bounds between 1\% [17] and 10\% [18] (see also [19]). We will denote the upper bound as $f$.

We will assume that the fraction $f_{C D M}$ of the dark matter is cold, and the rest hot. There are two sources of fluctuations, the conventional adiabatic perturbations (e.g. produced by quantum fluctuations during inflation) and the cosmic strings. We will assume that the cosmic strings are not correlated with the inflationary perturbations. In this case, the cross terms (between the string fluctuations and the adiabatic perturbations) to the angular $\mathrm{CMB}$ power spectrum and to the mass power spectrum vanish, and the mass power spectrum $P(k)$ calculated in linear cosmological perturbation theory can be written as

$$
P(k)=(1-f) P_{a}(k)+f P_{C S}(k),
$$

where $P_{a}$ and $P_{C S}$ are the power spectra for a pure adiabatic model and a pure cosmic string model, respectively.

Since the tightest constraints on the fraction of hot dark matter come from the observed power spectrum of matter on the smallest scales for which linear perturbation theory is adequate and for which observational results are robust, we will in the following focus on these scales. For these scales, the contribution of hot dark matter to the matter power spectrum for adiabatic fluctuations is negligible, and thus

$$
P_{a}(k) \simeq f_{C D M}^{2} P_{0}(k),
$$

where $P_{0}$ is the power spectrum of the current concordance model (scale invariant adiabatic fluctuations in the $\Lambda \mathrm{CDM}$ model [31]). The result (2.2) is not exact for two reasons: first, since cold dark matter does cluster about the primordial perturbations, neutrino free streaming delays but does not totally prevent the clustering of neutrinos. This effect would lead to a spectrum larger than given in Eq. (2.2). On the other hand, the local clustering of the cold dark matter occurs as if the Universe were slightly open. This effect tends to reduce the spectrum from the result (2.2). However, both of these effects are small if the fraction of hot dark matter is not too large. Even if we use the exact transfer function of adiabatic fluctuations [20], the final result does not change significantly.

On small scales, the transfer function for a model with cosmic strings and cold dark matter is different from the transfer function in the concordance model [21]. The transfer function is, in fact, much larger. Thus, as noted in [21], a pure cosmic string model which is $\sigma_{8}$-normalized (i.e. normalized such that the power spectrum on a length scale of $8 h^{-1} \mathrm{Mpc}$ agrees with observations) produces too much small scale structure. Hot dark matter clustering onto cosmic string loops, on the other hand, is delayed but not prevented. Thus, the hot dark matter power spectrum in a cosmic string model on small scales is not exponentially suppressed. However, the hot dark matter cosmic string power spectrum on small scales is much smaller than the cold dark matter power spectrum, so that to a good approximation we can use

$$
P_{C S}(k) \simeq f_{C D M}^{2} P_{(0, C S)},
$$


where $P_{(0, C S)}$ denotes the power spectrum in a pure cosmic string model with cold dark matter and the current concordance parameters.

The question we would like to ask is whether the contribution of cosmic strings to the power spectrum, given by Eqs. (2.1) and (2.3) is large enough to compensate for the loss in small scale power from the adiabatic fluctuations [given by Eq. (2.2)] if $f_{C D M}<1$ and to render the theory compatible with observations. More specifically, we are interested in finding the smallest value of the cold dark matter fraction $f_{C D M}$ for which the theoretical power spectrum $P(k)$ exceeds the observational result $P_{o b s}(k)$, i.e.

$$
P(k)>P_{o b s}(k)
$$

on the smallest scales for which high quality observational results exist and for which we can trust the results of linearized perturbation analysis, namely $k=0.2 \mathrm{~h} \mathrm{Mpc}^{-1}$ [22].

The results of [23] indicate that on scales of $k$ $=0.2 \mathrm{~h} \mathrm{Mpc}^{-1}$ the cosmic string cold dark matter power spectrum $P_{(0, C S)}$ does not exceed the adiabatic cold dark matter spectrum $P_{0}(k)$ by more than a factor of 2 . In fact, even on scales as low as $1 h^{-1} \mathrm{Mpc}$, the difference is not more than a factor of 10. The results of [23] are for models without a cosmological constant. However, as demonstrated in [24], on the smaller scales of relevance here, the matter power spectrum does not depend on the value of the cosmological constant in a significant way, and thus we can make use of the results of [23]. On the other hand, it is well known that the perturbations seeded by strings are isocurvature in nature (see e.g. [11]). For isocurvature fluctuations, the CMB temperature anisotropies on large scales are given by

$$
\frac{\delta T}{T}=2 \Phi
$$

where $\Phi$ is the relativistic gravitational potential (see e.g. [25]) which determines the magnitude of the mass perturbations. This is to be compared with

$$
\frac{\delta T}{T}=\frac{1}{3} \Phi,
$$

in the case of adiabatic fluctuations. Thus, if we normalize the power spectrum by the CMB anisotropies on large angular scales, the cosmic string power spectrum on large scales is a factor of 36 smaller than the power spectrum for adiabatic fluctuations. Hence, we see that on scales relevant to the large-scale structure, the decrease in the matter power spectrum which results from the addition of strings as a secondary source of cosmological fluctuations far exceeds the increase which is obtained by the change in the transfer function. Using the optimistic value 2 for the increase in the power due to the change in the transfer function on the relevant scale of $k=0.2 \mathrm{~h} \mathrm{Mpc}^{-1}$, we conclude that in a model with both cosmic string and adiabatic scale-invariant fluctuations

$$
\begin{aligned}
P(k) & =(1-f) P_{a}(k)+f P_{C S}(k) \\
& \leqslant(1-f) f_{C D M}^{2} P_{0}(k)+f f_{C D M}^{2} \frac{2}{36} P_{0}(k) \\
& <f_{C D M}^{2} P_{0}(k) .
\end{aligned}
$$

In a model with only adiabatic perturbations, the upper bound on the sum of the neutrino masses obtained by combining the large-scale structure and CMB data comes from demanding that

$$
P(k) \geqslant P_{l}(k)
$$

where $P_{l}(k)$ is the observational lower bound on the mass spectrum. Since in this case

$$
P(k) \simeq f_{C D M}^{2} P_{0}(k)
$$

and knowing that the $P_{0}(k)$ fits the date well, this gives a lower bound on $f_{C D M}$ which is equivalent to an upper limit on the sum of the neutrino masses.

Combining these last three equations, we conclude that the inclusion of a contribution of cosmic strings to the primordial power spectrum does not lead to a relaxation of the cosmological bounds on the sum of the neutrino masses.

\section{DISCUSSION}

In this paper we have shown that the current cosmological neutrino mass limits, which were derived under the assumption that the spectrum of cosmological perturbations is purely adiabatic, are robust against the addition of the maximal contribution which cosmic strings could make to the primordial power spectrum. A priori, this is not obvious, since cosmic string seeds survive neutrino free streaming and, therefore, clustering on small scales (scales which yield the tightest constraints on the neutrino masses) is not prevented but only delayed. However, if the power spectrum is normalized by the large-scale CMB anisotropies, then the contribution of strings to the matter power spectrum is suppressed by a factor of 36 since the primordial string perturbations are isocurvature instead of adiabatic.

To obtain the above result, we have assumed that the string-induced fluctuations and the adiabatic perturbations are statistically uncorrelated. If they were correlated, a cross term in the power spectrum would appear which would only be suppressed by a factor of 6 . We have also assumed that bias is unimportant, i.e. that the observed galaxy correlation function equals the calculated matter power spectrum. However, in a cosmic string model we expect significant biasing (in particular in a model in which the dark matter has a significant component of hot matter). However, in the absence of a better understanding of the dynamics of cosmic strings (in particular whether string loops [26] or string wakes [27-29] are dominant), no firm conclusions can be drawn.

Even if it turns out that the addition of a cosmic string component to the primordial power spectrum does not change the cosmological neutrino bounds, this addition will 
be important for many cosmological issues, in particular for early structure formation [30] and reionization [17].

\section{ACKNOWLEDGMENTS}

We are grateful to Guy Moore for interesting conversations at the beginning of this project. R.B. acknowledges the hospitality of the McGill Physics Department and the Fields Institute during the course of this work. At Brown, this work was supported in part by the U.S. Department of Energy under Contract DE-FG02-91ER40688, TASK A. M.Y. was partially supported by the Japanese Grant-in-Aid for Scientific Research from the Ministry of Culture, Sports, Science, and Technology. A.M. acknowledges support from CITA and NSERC.
[1] A.Y. Smirnov, Nucl. Phys. B (Proc. Suppl.) 118, 87 (2003); J.N. Bahcall, M.C. Gonzalez-Garcia, and C. Pena-Garay, J. High Energy Phys. 02, 009 (2003); 08, 014 (2001).

[2] D.N. Spergel et al., Astrophys. J., Suppl. 148, 175 (2003); S. Hannestad, J. Cosmol. Astropart. Phys. 05, 004 (2003); S.W. Allen, R.W. Schmidt, and S.L. Bridle, Mon. Not. R. Astron. Soc. 346, 593 (2003); V. Barger, D. Marfatia, and A. Tregre, hep-ph/0312065.

[3] L. Verde et al., Astrophys. J., Suppl. 148, 195 (2003); SDSS Collaboration, M. Tegmark et al., Phys. Rev. D (to be published), astro-ph/0310723.

[4] J.R. Bond, G. Efstathiou, and J. Silk, Phys. Rev. Lett. 45, 1980 (1980); G. Bisnovatyi-Kogan and I. Novikov, Astron. Zh. 57, 899 (1980).

[5] A.D. Linde and V. Mukhanov, Phys. Rev. D 56, 535 (1997).

[6] R. Jeannerot, Phys. Rev. D 53, 5426 (1996); R. Jeannerot, J. Rocher, and M. Sakellariadou, ibid. 68, 103514 (2003).

[7] S. Sarangi and S.H.H. Tye, Phys. Lett. B 536, 185 (2002); N.T. Jones, H. Stoica, and S.H.H. Tye, ibid. 563, 6 (2003).

[8] R.H. Brandenberger, N. Kaiser, D.N. Schramm, and N. Turok, Phys. Rev. Lett. 59, 2371 (1987).

[9] R.H. Brandenberger, N. Kaiser, and N. Turok, Phys. Rev. D 36, 2242 (1987).

[10] C.L. Bennett et al., Astrophys. J., Suppl. 148, 1 (2003).

[11] J. Traschen, N. Turok, and R.H. Brandenberger, Phys. Rev. D 34, 919 (1986).

[12] U.L. Pen, U. Seljak, and N. Turok, Phys. Rev. Lett. 79, 1611 (1997).

[13] J. Magueijo, A. Albrecht, D. Coulson, and P. Ferreira, Phys. Rev. Lett. 76, 2617 (1996).
[14] A. Albrecht, R.A. Battye, and J. Robinson, Phys. Rev. Lett. 79, 4736 (1997).

[15] B. Allen, R.R. Caldwell, S. Dodelson, L. Knox, E.P.S. Shellard, and A. Stebbins, Phys. Rev. Lett. 79, 2624 (1997).

[16] L. Perivolaropoulos, Astrophys. J. 451, 429 (1995).

[17] P.P. Avelino and A.R. Liddle, astro-ph/0305357.

[18] L. Pogosian, S.H.H. Tye, I. Wasserman, and M. Wyman, Phys. Rev. D 68, 023506 (2003).

[19] M. Endo, M. Kawasaki, and T. Moroi, Phys. Lett. B 569, 73 (2003).

[20] D.J. Eisenstein and W. Hu, Astrophys. J. 511, 5 (1997).

[21] A. Albrecht and A. Stebbins, Phys. Rev. Lett. 68, 2121 (1992).

[22] S. Hannestad, J. Cosmol. Astropart. Phys. 05, 004 (2003).

[23] A. Albrecht and A. Stebbins, Phys. Rev. Lett. 69, 2615 (1992).

[24] P.P. Avelino, E.P.S. Shellard, J.H.P. Wu, and B. Allen, Phys. Rev. Lett. 81, 2008 (1998); J.H.P. Wu, P.P. Avelino, E.P.S. Shellard, and B. Allen, Int. J. Mod. Phys. D 11, 61 (2002).

[25] V.F. Mukhanov, H.A. Feldman, and R.H. Brandenberger, Phys. Rep. 215, 203 (1992).

[26] N. Turok and R.H. Brandenberger, Phys. Rev. D 33, 2175 (1986).

[27] J. Silk and A. Vilenkin, Phys. Rev. Lett. 53, 1700 (1984).

[28] T. Vachaspati, Phys. Rev. Lett. 57, 1655 (1986).

[29] A. Stebbins, S. Veeraraghavan, R.H. Brandenberger, J. Silk, and N. Turok, Astrophys. J. 322, 1 (1987).

[30] R. Moessner and R.H. Brandenberger, Mon. Not. R. Astron. Soc. 280, 797 (1996).

[31] We will consider the same background cosmological parameters $\Lambda, h$, etc., as in this concordance model. As emphasized in [19], the addition of cosmic strings to the model may yield a slightly different best fit value for the parameters. We will return to this issue in future work. 